\title{
Blocking Toll-like receptor 9 attenuates bleomycin-induced pulmonary injury
}

\author{
Badr Alzahrani ${ }^{1}$, Mohamed M. S. Gaballa ${ }^{2}$, Ahmed A. Tantawy ${ }^{2}$, Maha A. Moussa ${ }^{3}$, Salma A. Shoulah ${ }^{4}$, Said M. Elshafae \\ 'Department of Clinical Laboratory Sciences, College of Applied Medical Sciences, Jouf University, Sakaka, Saudi Arabia; \\ 2Department of Pathology, Faculty of Veterinary Medicine, Benha University, Tukh; \\ ${ }^{3}$ Department of Statistics, Faculty of Commerce, Benha University, Benha; \\ ${ }^{4}$ Department of Animal Medicine (Infectious Diseases), Faculty of Veterinary Medicine, Benha University, Tukh, Egypt
}

\begin{abstract}
Background: Acute respiratory distress syndrome (ARDS) is one of the most common complications in coronavirus disease 2019 patients suffering from acute lung injury (ALI). In ARDS, marked distortion of pulmonary architecture has been reported. The pulmonary lesions in ARDS include hemodynamic derangements (such as alveolar edema and hemorrhage), vascular and bronchiolar damage, interstitial inflammatory cellular aggregations, and eventually fibrosis. Bleomycin induces ARDS-representative pulmonary damage in mice and rats; therefore, we used bleomycin model mice in our study. Recently, Toll-like receptor 9 (TLR9) was implicated in the development of ARDS and ALI. Methods: In this study, we evaluated the efficiency of a TLR9 blocker (ODN2088) on bleomycin-induced pulmonary damage. We measured the apoptosis rate, inflammatory reaction, and fibroplasia in bleomycin- and bleomycin + ODN2088-treated mice. Results: Our results showed a significant amelioration in bleomycin-induced damage to pulmonary architecture following ODN2088 treatment. A marked decrease in pulmonary epithelial and endothelial apoptosis rate as measured by cleaved caspase-3 expression, inflammatory reaction as indicated by tumor necrosis factor $\alpha$ expression, and pulmonary fibrosis as demonstrated by Van Gieson staining and $\alpha$-smooth muscle actin immunohistochemistry were observed following ODN2088 treatment. Conclusions: All these findings indicate that blocking downstream TLR9 signaling could be beneficial in prevention or mitigation of ARDS through hemodynamic derangements, inflammation, apoptosis, and fibrosis.
\end{abstract}

Key Words: TLR9; ODN2088; Bleomycin; Acute respiratory distress syndrome; Fibrosis

Received: November 2, 2021 Revised: December 4, 2021 Accepted: December 27, 2021

Corresponding Author: Said M. Elshafae, PhD, Department of Pathology, Faculty of Veterinary Medicine, Benha University, Moshtohor, Tukh, Qalyobiya 13736, Egypt

Tel: +20-1435-363-5372, E-mail: Said.Alshafey@fvtm.bu.edu.eg

In December 2019, several patients in China were infected with a novel coronavirus known as severe acute respiratory syndrome coronavirus 2, the etiological agent underlying acute respiratory disease named coronavirus disease 2019 (COVID-19) [1]. Based on clinical and radiologic data, lung injury is one of the most common complications of COVID-19. In about $20 \%$ of COVID-19 patients, acute lung injury (ALI) rapidly progresses into acute respiratory distress syndrome (ARDS) [2]. ARDS development includes acute, subacute, and chronic phases [3]. The acute phase is mainly characterized by alveolar and interstitial edema, endothelial and epithelial damage, and aggregations of inflammatory cells and red blood cells (RBCs) in the alveoli. The predominant changes in the subacute phase are pulmonary edema clearance, type II alveolar pneumocyte hyperplasia, fibroblastic proliferation, and collagen deposition [3]. The chronic phase of ARDS involves infiltrations of alveolar macrophages in the alveoli and increased fibrosis. The bleomycin-induced lung injury model is a well-characterized model of pulmonary damage, edema, inflammation, and eventual fibrosis that closely resembles the ARDS development pathway [4]. The bleomycin mouse model is an excellent research tool to investigate ARDS due to strong similarities regarding the cells, mediators, and signaling pathways that contribute to pathogenesis.

Bleomycin, generated by the bacterium Streptomyces verticillus, is a glycosylated linear non-ribosomal peptide antibiotic. Since bleomycin has potent antitumorigenic properties, it is used as a chemotherapeutic agent against many cancers, including squamous cell carcinomas of the cervix, esophagus, and head and neck; 
germ cell tumors; and both Hodgkins and non-Hodgkins lymphoma [5-7]. Bleomycin causes direct cellular damage by disrupting DNA strands, creating free radicals, and inducing oxidative stress [8,9]. Upon damage, the affected cells excrete uric acid and many other factors that initiate aggregation of alveolar macrophages and release of pro-inflammatory cytokines. During the first week after bleomycin administration, marked elevation of pro-inflammatory cytokines (tumor necrosis factor $\alpha$ [TNF- $\alpha$ ], interleukin [IL]-1, and IL-6) was reported. Upregulation of profibrotic markers (transforming growth factor $\beta 1$ [TGF- $\beta 1$ ], fibronectin, and collagen) was seen by day 14 . By the second to third weeks, patchy fibrotic areas with extracellular matrix (ECM) deposition, mainly in the forms of fibronectin and collagen I, are evident in pulmonary tissue [10].

Toll-like receptors (TLRs) have a vital role in the innate immune system [11-13]. Hyperactivation of TLRs disrupts immune system homeostasis, resulting in extensive pro-inflammatory cytokine production that has a key role in many autoimmune and inflammatory diseases [14]. Among other TLRs, TLR9 was found to promote the release of pro-inflammatory mediators such as TNF- $\alpha$ and IL- $1 \beta$ in ALI and is implicated in several chronic inflammatory pulmonary conditions [15]. Recent studies showed that TLR9 contributes to the development and worsening of ARDS and ALI in COVID-19 patients [16-18]. Furthermore, TLR9 induction was found to activate blood coagulation in humans and mice $[19,20]$. All these findings suggest that blocking the TLR signaling pathway could be an effective therapeutic strategy for mitigating the clinical presentation of ARDS and ALI in COVID-19 patients.

CpG-oligodeoxynucleotide ODN is an unmethylated, short, single-stranded synthetic DNA molecule that acts as a TLR9 ligand [21]. Activation of TLR9 using the ODN ligand promotes neutrophilic infiltration, pulmonary edema, and systemic inflammation $[15,16]$. The oligonucleotide ODN2088 (a DNA sequence) inhibits the activity of TLR9 and its downstream signaling effectors by disturbing the co-localization of unmethylated CPG di-nucleotides with TLR9 [22]. A previous study showed that ODN2088 inhibits the liver inflammation and fibrosis induced by CCl4 [23]. In the present study, a bleomycin-induced lung injury mouse model was used to evaluate the impact of TLR9 inhibition by ODN2088 on the lung damage, inflammation, and fibrosis commonly seen in ARDS in COVID-19 patients.

\section{MATERIALS AND METHODS}

\section{Chemicals}

CpG ODN2088 was purchased from Invitrogen (Carlsbad, CA, USA). ODN 2088 is a TLR9 antagonist with 15 base pairs (5'-tcctggcggggaagt-3'). Bleomycin sulfate (Celon Laboratories Pvt. Ltd., Hyderabad, India) was dissolved in sterile saline solution for injection.

\section{In vivo experimental design}

Swiss Albino mice were purchased from the Facility of Laboratory Animals at the Facility of Veterinary Medicine, Benha University, Egypt. All animal experiments were conducted according to protocols approved by the Benha University Committee of Bioethics in Egypt. Mice were maintained according to the National Institutes of Health standards established in the "Guidelines for the Care and Use of Laboratory Animals." Forty adult 6-8-weekold male Swiss Albino mice (22-30 g) were used in this study. The mice were subdivided equally into four groups. In the first group (control), mice were injected intraperitoneally (IP) with saline (vehicle). In the second group (bleomycin), mice were injected with bleomycin IP (2 mg/mouse) on days 1,8 , and 15 . In the third group (ODN2088), the mice were injected IP with CpG ODN2088 $(50 \mu \mathrm{g} / 20 \mathrm{~g} \mathrm{BW}$, re-suspended in endotoxin-free water) daily until the day of sacrifice. In the fourth group (bleomycin + ODN2088) (BLEO/ODN2088), the mice received both bleomycin (2 mg/mouse) and ODN2088 (50 $\mu \mathrm{g} / 20 \mathrm{~g} \mathrm{BW})$.

\section{Histopathology}

The mice were euthanized after 30 days by cervical dislocation, and pulmonary tissue specimens were collected during necropsy. Lung specimens were fixed in 10\% neutral-buffered formalin at room temperature for 72 hours, embedded in paraffin, cut in 4 $\mu \mathrm{m}$ sections, and stained with hematoxylin and eosin. Histological images of the slides were collected using a Nikon Eclipse E800 microscope (Melville, NY, USA) equipped with a camera and were analyzed using Fiji ImageJ software (ImageJ 1.51u, National Institutes of Health, Bethesda, MD, USA).

\section{Van Gieson staining}

Pulmonary tissue sections were washed with tap water and incubated in picrofuchsin solution (1\% acid fuchsin in aqueous saturated picric acid) for 2 minutes. Sections were dehydrated and mounted using DPX mounting agent (Thermo Fisher Scientific, Leicester, UK). Upon staining, collagen fibers appear pink to red, while muscle appears yellowish. The area of positive collagen or 
elastic fibers was calculated compared to the total area of the tissue section.

\section{Immunohistochemistry}

The 5- $\mu \mathrm{M}$-thick paraffin-embedded tissue sections were preheated and dewaxed in xylene followed by dehydration in ethanol. The tissue sections were incubated with antigen retrieval solution (Dako, Carpinteria, CA, USA) for 40 minutes using a steamer (Black \& Decker HS1000, Newark, DE, USA) followed by a cooling step (for 30 minutes at room temperature). The tissue sections were treated with $3 \%$ hydrogen peroxide to block endogenous peroxidase activity and washed, and $10 \%$ bovine serum albumin was added to block non-specific immunoreactivity. The slides were incubated overnight with either anti-cleaved caspase-3 (1:400, Cat \#Asp175, Cell Signaling Technology, Danvers, MA, USA), anti-TNF- $\alpha$ (1:400, NBP1-19532, Novus, Centennial, CO, USA), or anti- $\alpha$-smooth muscle actin ( $\alpha$-SMA; $1: 50$, clone 1A4, Dako) primary antibodies. On the next day, slides were washed three times and incubated with the corresponding biotinylated secondary antibody diluted in phosphate buffered saline (PBS) for 1 hour at $25^{\circ} \mathrm{C}$, followed by three PBS washes. The slides were incubated with diaminobenzidine tetrahydrochloride solution for 10 minutes. The slides were washed three times in distilled water and counter stained with hematoxylin. Tissue sections were finally dehydrated using graded alcohols and xylene and then covered with a glass slip [24]. DAB staining (brown color) was recorded as a positive result. Positivity was counted as number of positive cells/number of total cells. ImageJ ver. $1.48(\mathrm{Na}-$ tional Institutes of Health, Bethesda, MD, USA) was used to calculate the percent positivity in all the pulmonary tissues.

\section{Statistical analysis}

All data collected are displayed as mean \pm standard deviation. Student's unpaired t-test was used to analyze the difference in positivity between bleomycin and BLEO/ODN2088 groups using GraphPad Prism ver. 6.03 (La Jolla, CA, USA). Differences denoted by $\mathrm{p} \leq .05$ were considered statistically significant.

\section{RESULTS}

\section{ODN2088 restored pulmonary architecture distorted by bleomycin}

In control mice, almost all the lung sections had normal pulmonary architecture with clear empty alveoli and thin interalveolar septa (Fig. 1A). The alveoli were lined with flat (type I pneumocytes) and cuboidal cells projecting into the alveolar lumen (type II pneumocytes). Patent bronchioles lined with simple columnar epithelium were also evident in most sections. Most lungs in ODN2088-treated mice exhibited normal histological appearance with thin interalveolar septa and patent alveoli. In a few lungs, mild alveolar pneumocyte hyperplasia was observed (Fig. 1B). Alveolar emphysema was seen occasionally in some pulmonary areas.

In bleomycin-treated mice, pulmonary architecture was severely distorted in a patchy pattern. Multifocal thickening of interalveolar septa and alveolar collapse were the most pronounced microscopic findings in all the lung sections. Meanwhile, most other alveoli were over-inflated (emphysematous). Alveolar septal thickening was mainly due to moderate to severe mononuclear inflammatory cell infiltration in the interalveolar space (Fig. 1CE). Moderate type II pneumocyte hyperplasia with reactive atypia and fibroplasia was evident in several pulmonary areas. Focal alveolar histiocytosis characterized by accumulation of foamy macrophages in the alveoli was also prominent in some lung sections. Fine eosinophilic fibrin threads were seen in some alveoli. In many pulmonary sections, hyperplasia of the bronchiolar epithelium was reported. Some hyperplastic bronchioles had cytoplasmic vacuolation and prominent enlarged nuclei in their epithelia, while others suffered from necrosis of their lining epithelia with the presence of desquamated intraluminal cells (Fig. 1F-H). Inflammatory peribronchial cell aggregations and fibrosis were also reported in some foci (Fig. 1H-J). Congestion of peribronchiolar blood vessels and interalveolar capillaries, edema, and hemorrhage were the main vascular changes in bleomycin-treated group. There were extensive perivascular inflammatory cell infiltrates, including lymphocytes and macrophages, affecting some large pulmonary blood vessels along with extravasated RBCs in nearby alveoli (Fig. 1J). Fibrinoid necrosis was also observed in the walls of a few large pulmonary blood vessels in some sections (Fig. 1J). Other vascular alterations included disorganization and hypertrophy of tunica media, fibrous remodeling, and occasional pulmonary vein thrombosis (Fig. 1K). Multifocally, some alveoli were filled with extravasated RBCS, homogenous eosinophilic fluid, and siderophages. In some mice, pleura thickened by edema, fibrin deposition, and occasional infiltration of lymphocytes and macrophages and, rarely, neutrophils were seen (Fig. 1L).

Apparent amelioration of bleomycin-induced lung distortion, marked by distinct restoration of lung architecture, was noticed in the BLEO/ODN2088-treated group compared to the bleomycin-treated group. Most bronchiolar epithelia had mild goblet cell hyperplasia or minimal degenerative/necrotic changes (Fig. $1 \mathrm{M})$. Most alveoli were devoid of fluids, RBCs, fibrin threads, 

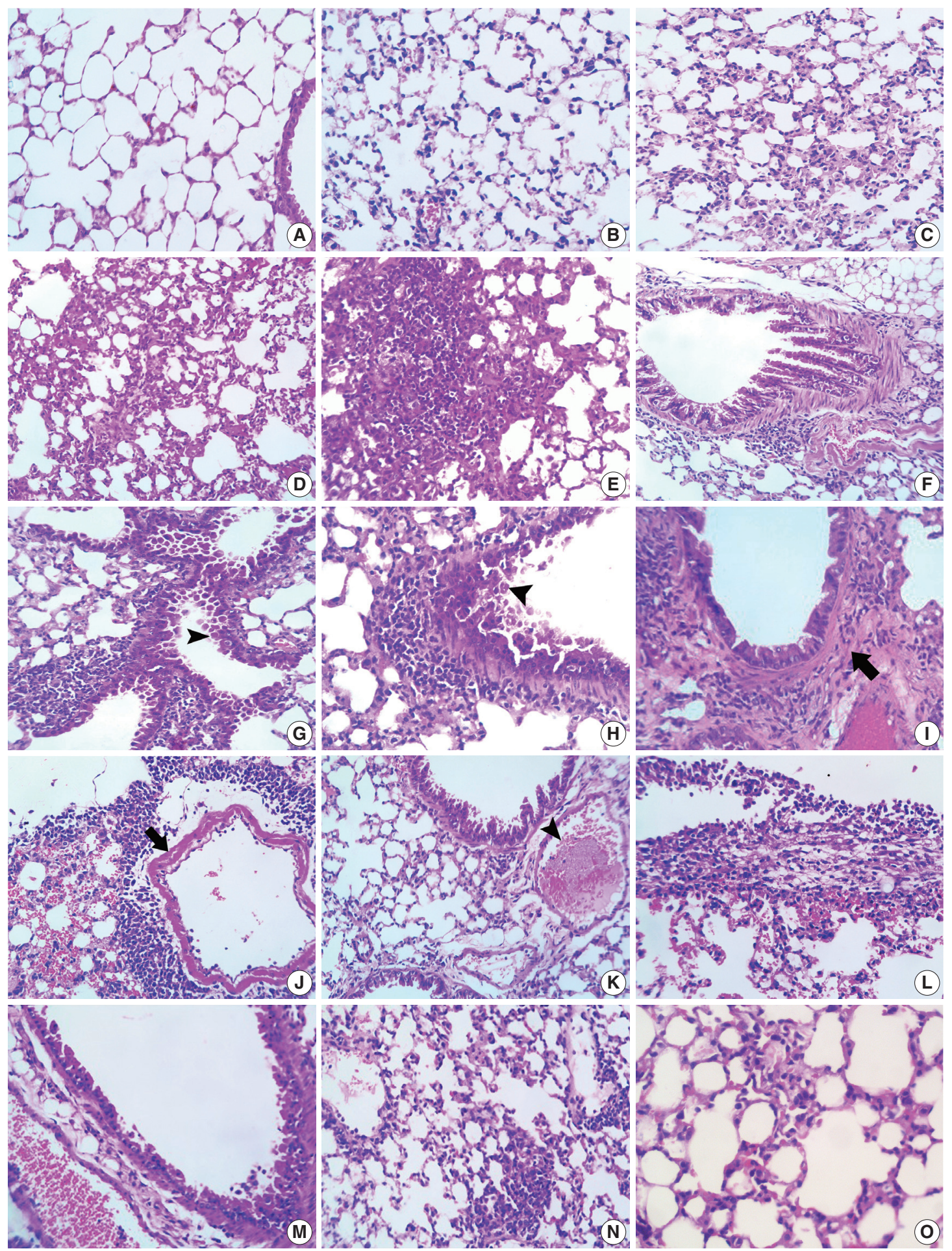

Fig. 1. Representative lung photomicrographs in control- (A), ODN2088- (B), bleomycin- (C-L), and bleomycin + ODN2088 (BLEO/ ODN2088)-treated mice (M-O). (A) Normal respiratory bronchioli and alveoli in the control group. (B) Mild hyperplasia of alveolar pneumocytes in the lung of an ODN2088-treated mouse. (C-L) Lung sections of mice treated with bleomycin, showing focal to confluent, moderate, to severe aggregation of inflammatory cells in the interalveolar septa (C-E), hyperplastic bronchiolar epithelia (F), peribronchial inflammatory aggregations and necrosis of bronchiolar epithelia (G, H, arrowhead), peribronchiolar fibroplasia (I, arrow), perivascular (arrow) inflammatory cellular infiltration and intra-alveolar hemorrhage $(\mathrm{J})$, thrombosis in a pulmonary blood vessel (K, arrowhead), and pleural thickening (L). (M-O) Lungs of BLEO/ODN2088-treated mice showing necrosis in a few bronchiolar epithelia (M), small focal inflammatory aggregation in the interstitial space (N), and congested interalveolar blood capillaries (O). 
and inflammatory cells. There was no to mild thickening of interlobular septa due to mild mononuclear cell aggregations, congestion of interalveolar blood capillaries, and mild interlobular edema (Fig. 1N, O). Overinflation of alveoli with mild edema was occasionally seen.

\section{ODN2088 reduced bleomycin-induced pulmonary fibrosis}

Van Gieson staining and $\alpha$-SMA immunohistochemistry were used to assess the antifibrotic effects of ODN2088 against pulmonary fibrosis induced by bleomycin. Van Gieson-stained sections demonstrated control and ODN2088-treated mice to have only a thin layer of pink-stained collagen fibers in the tunica adventitia of pulmonary blood vessels and bronchioles. In bleomycin-treated mice, on the other hand, multifocal areas of tightly packed pink-stained collagen fibers were seen around bronchioles and blood vessels (Fig. 2A, B). Multifocal red-stained collagen fibers were also seen in the interalveolar and interlobular spaces. The pulmonary tissues of the BLEO/ODN2088 group stained with Van Gieson stain were nearly similar to those of the control group (Fig. 2C). Compared to the bleomycin group, the area of Van Gieson-stained collagen fibers was significantly smaller in the BLEO/ODN2088-treated group (Fig. 2D)

\section{ODN2088 mitigated bleomycin-induced myofibroblast proliferation in pulmonary tissue}

To further confirm the findings of Van Gieson staining, we used $\alpha$-SMA as an immunohistochemical marker for myofibroblast proliferation and fibrosis. There was very weak $\alpha$-SMA immunos-
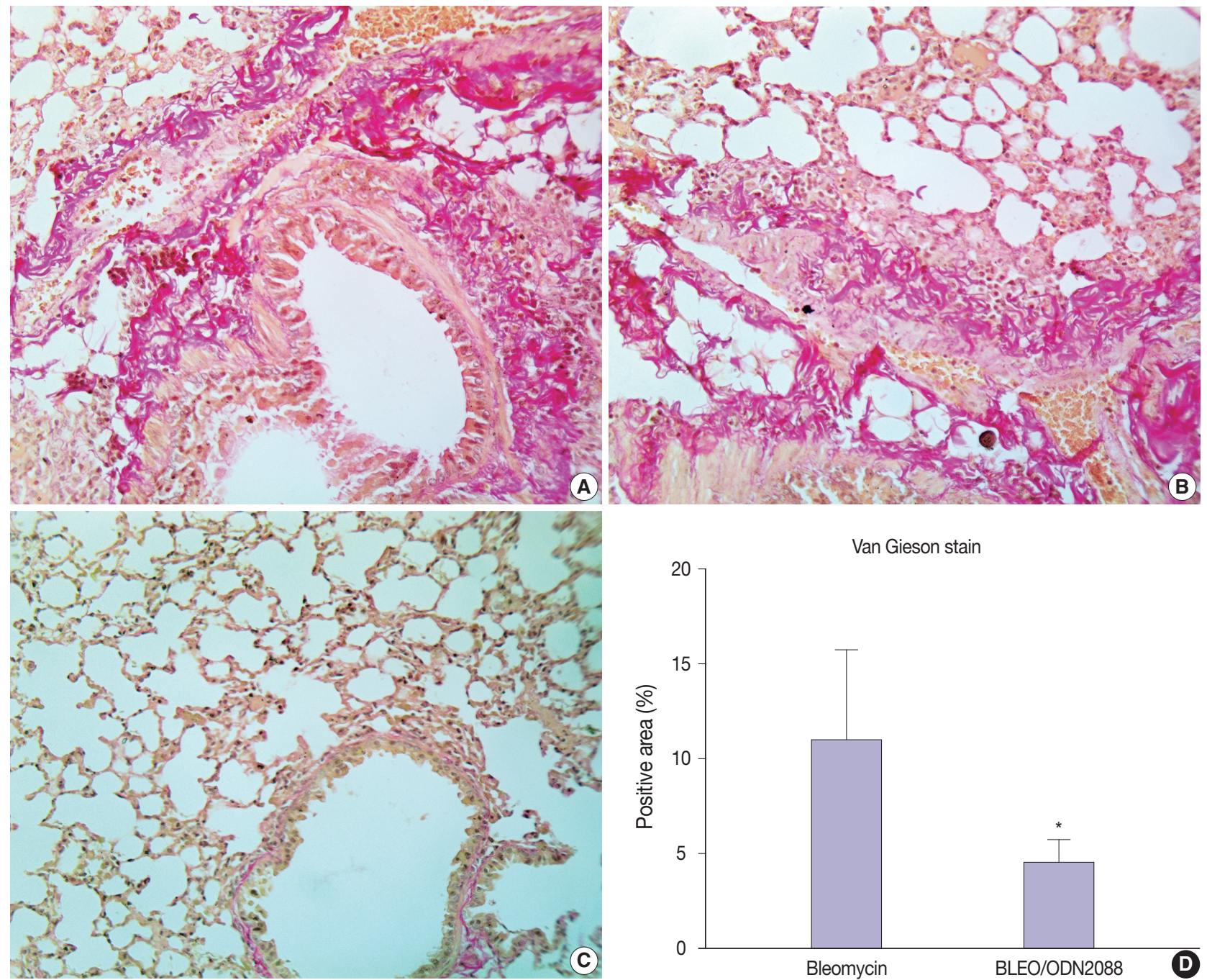

Fig. 2. Photomicrographs $(A-C)$ and statistical analysis (D) of Van Gieson staining of pulmonary tissues in bleomycin- (A, B) and bleomycin+ODN2088 (BLEO/ODN2088)-treated mice (C). Data in the bar graph (D) represent mean \pm standard deviation, and significant differences $(\mathrm{p} \leq .05)$ are indicated by an asterisk. 
taining in the pulmonary tissues of control and ODN2088-treated mice. In bleomycin-treated mice, a marked increase in $\alpha$-SMA protein expression (evidenced by brown DAB staining) was seen multifocally in the alveoli, interalveolar septa, and peribronchial connective tissue (Fig. 3A, B). ODN2088 reduced myofibroblast proliferation in the pulmonary tissues of the BLEO/ODN2088treated group, demonstrated by a marked decline in $\alpha$-SMA expression (Fig. 3C, D). Focal areas with mild $\alpha$-SMA staining were seen in the interalveolar septa of some lungs in this group.

\section{ODN2088 lowered the apoptosis rate in}

\section{BLEO/ODN2088-treated pulmonary tissues}

Staining for cleaved caspase- 3 was used to assess the effect of
ODN2088 on apoptosis caused by bleomycin. There was minimal cleaved caspase- 3 expression in the control- and ODN2088treated groups. Extensive damage to pulmonary tissues was seen in bleomycin-treated mice, where there was a marked increase in cleaved caspase- 3 expression in many alveolar pneumocytes, bronchiolar epithelial cells, and vascular endothelial cells (Fig. 4A, B). ODN2088 mitigated the pulmonary toxicity of bleomycin in the BLEO/ODN2088 group. The ameliorative effects of ODN2088 were pronounced in bronchiolar epithelium and endothelial cells (Fig. 4C). Compared to the bleomycin group, there were significantly fewer cleaved caspase-3-positive cells in the BLEO/ODN2088-treated group (Fig. 4D).
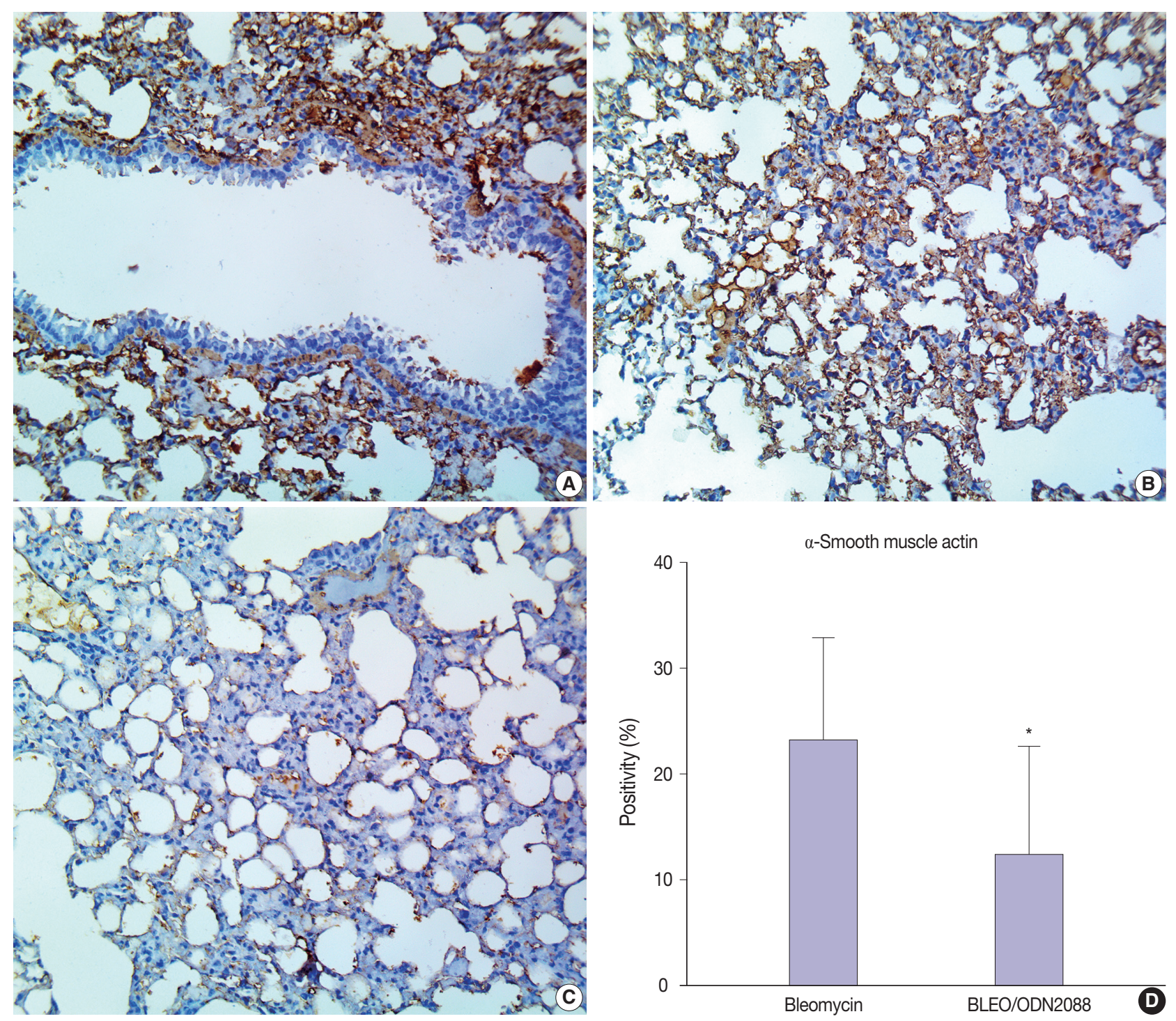

Fig. 3. Immunohistochemical photomicrographs (A-C) and statistical analysis (D) of $\alpha$-smooth muscle actin $(\alpha-S M A)$ expression in the pulmonary tissues of bleomycin- (A, B) and bleomycin+ODN2088 (BLEO/ODN2088)-treated mice (C). Data in the bar graph (D) represent mean \pm standard deviation, and significant differences $(\mathrm{p} \leq .05)$ are indicated by an asterisk. 
ODN2088 mitigated the bleomycin-induced inflammatory reaction

To assess the potential inhibitory effects of ODN2088 on the bleomycin-induced inflammatory reaction, we evaluated TNF- $\alpha$ expression in pulmonary tissues. Null to mild TNF- $\alpha$ expression was seen in the pulmonary tissues of control and ODN2088-treated mice. In bleomycin-treated mice, extensive TNF- $\alpha$ expression was observed in alveolar macrophages and the inflammatory cellular aggregations in the interalveolar space and peribronchiolar, perivascular, and pleural connective tissue (Fig. 5A, B). ODN2088 markedly reduced TNF- $\alpha$ expression in pulmonary tissues in the BLEO/ODN2088-treated group. There was mild cellular TNF- $\alpha$ expression in the interalveolar septa and peribronchiolar connective tissue (Fig. 5C). In only one mouse, moderate to severe focal TNF- $\alpha$ expression was observed in the inflammatory cells in the interalveolar septa. The number of TNF- $\alpha$ positive cells was much lower in the BLEO/ODN2088-treated group compared to the bleomycin group (Fig. 5D).

\section{DISCUSSION}

Pulmonary damage is one of the most serious complications of diffuse lung diseases, such as idiopathic pulmonary fibrosis, interstitial lung disease, and ARDS [25-29]. Persistent pulmo-
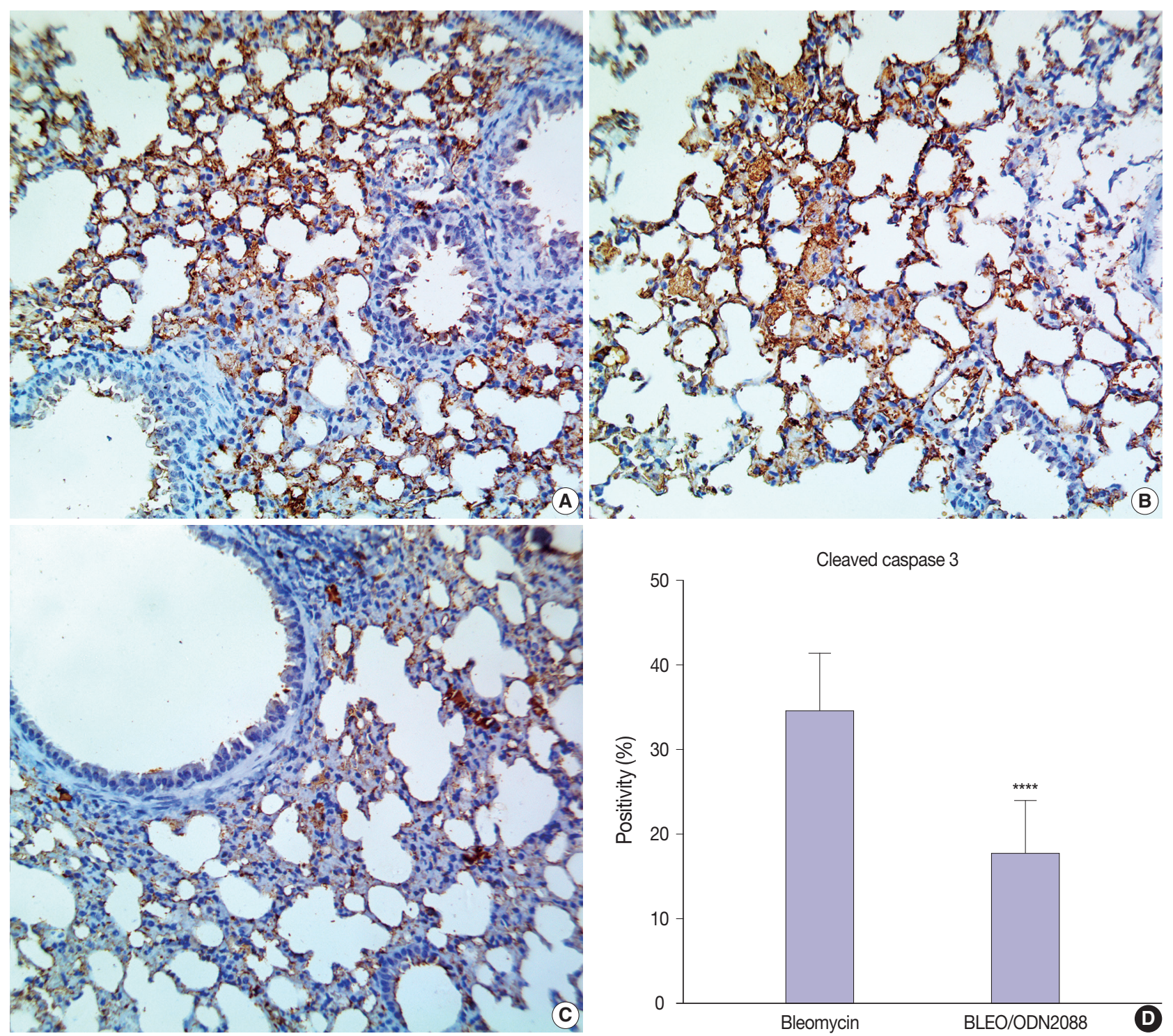

Cleaved caspase 3

Fig. 4. Cleaved caspase-3 (CC3) immunohistochemical photomicrographs (A-C) and statistical analysis of CC3-positive cells (D) in the pulmonary section of bleomycin- and bleomycin+ODN2088 (BLEO/ODN2088)-treated mice. Data in the bar graphs (D) represent mean \pm standard deviation, and significant differences $(p<.001)$ are indicated by four asterisks. 

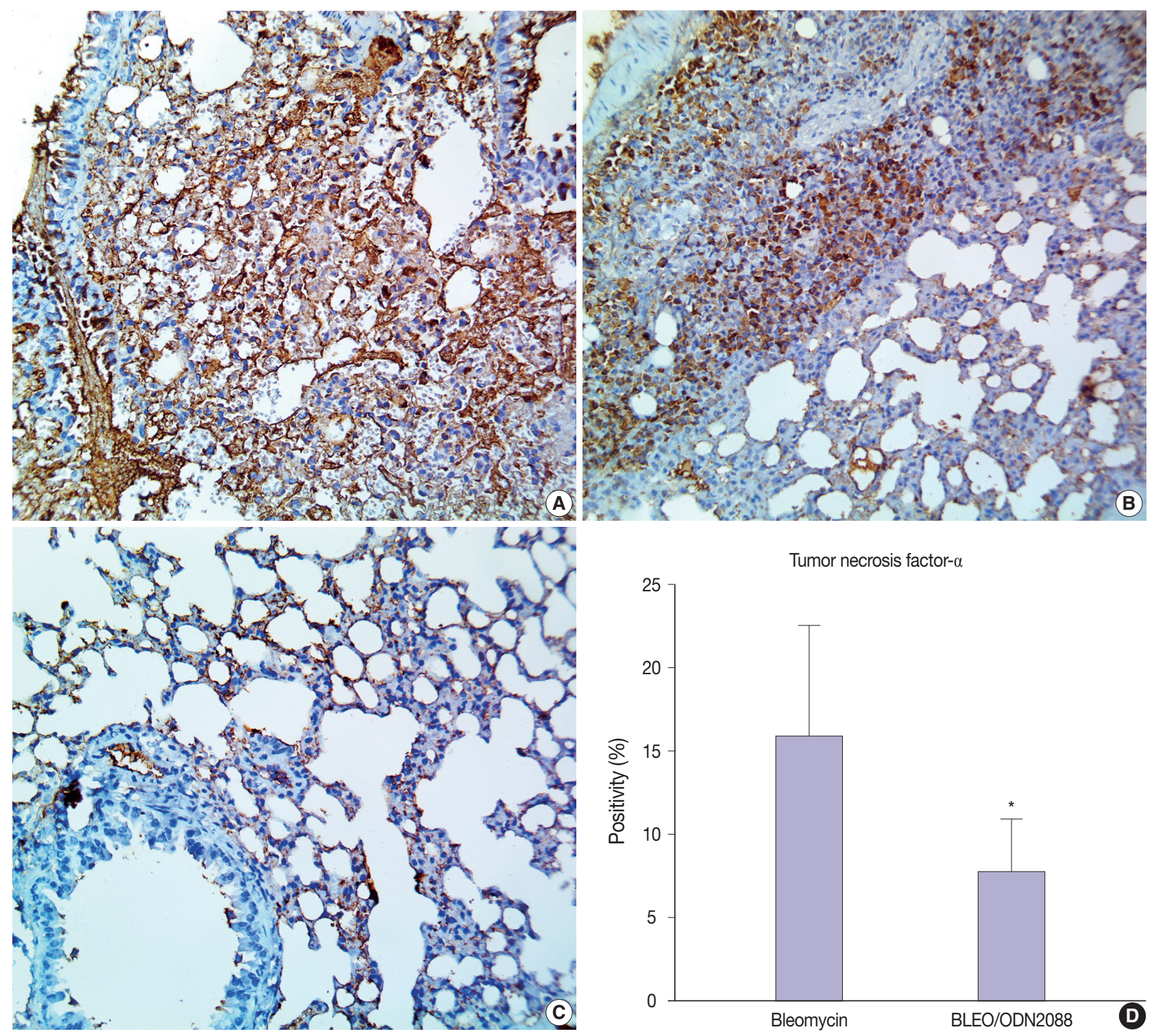

Fig. 5. Tumor necrosis factor $\alpha(T N F-\alpha)$ immunohistochemical photomicrographs (A-C) and analysis of TNF- $\alpha$ positivity (D) in the lung sections of bleomycin- and bleomycin + ODN2088 (BLEO/ODN2088)-treated mice. Data in the bar graph (D) represent mean \pm standard deviation, and significant differences $(\mathrm{p} \leq .05)$ are indicated by an asterisk.

nary damage has been shown to cause inflammation in the lungs, which eventually leads to pulmonary fibrosis [30]. Pulmonary fibrosis causes lung architecture distortion and excessive matrix deposition, which leads to respiratory insufficiency and death [31]. Many efforts are ongoing to alleviate the inflammatory process, lung injury, and pulmonary fibrosis to improve the quality of life in patients suffering from ARDS. There are several animal models that mimic the stages of pulmonary fibrosis in humans. In this research, we used bleomycin to induce lung fibrosis in mice since bleomycin-induced lung injury is a well-studied model of the changes seen in many pulmonary diseases, such as idiopathic pulmonary fibrosis, interstitial lung disease, and ARDS [26-29, 32]. Prior studies have indicated that ODN2088 is a potent antiinflammatory agent that halts downstream TLR9 pro-inflammatory pathways [33]. Therefore, the potential efficacy of ODN2088 in preventing bleomycin-induced lung damage, inflammation, and fibrosis was investigated in this study. Our study found that blocking TLR9 with ODN2088 prevented lung fibrosis in bleomycin-treated mice by reducing lung injury and inflammation.

Bleomycin initiates lung injury by generating reactive oxygen species that cause subsequent DNA damage [6]. In addition, bleomycin promotes protein oxidation and lipid peroxidation 
and induces an extreme inflammatory response and fibroblastic proliferation in animal models [34]. The fibrosis induced by bleomycin could be attributed to the cytokines secreted from alveolar macrophages following lung injury, such as TGF, IL-1, plateletderived growth factor, and macrophage inflammatory protein-1 [35]. These cytokines promote the proliferation of myofibroblasts and stimulate the production of abnormal extracellular matrix, leading to fibrosis [36]. Consistent with prior studies on the bleomycin mouse model, the lungs of bleomycin-treated mice in our study showed activation of alveolar macrophages, intense inflammatory response, and fibroblastic and myofibroblastic proliferation, which mimic the stages of lung injury/fibrosis in humans [27,37].

TLRs play an important role in recognizing viruses and triggering the innate immune system [38]. TLR stimulation triggers downstream signaling cascades, resulting in the release of proinflammatory cytokines, such as TNF- $\alpha$, IL- 6 , IL- 1 , and interferon type 1. It has been reported that, in severe cases of COVID-19, patients have marked elevation of inflammatory cytokines, such as IL-6, IL-8, IL-10, TNF- $\alpha$, interferon- $\gamma$, IL-2, and IL-4 [39,40]. All these findings indicate that targeting TLRs could mitigate the clinical presentation, outcomes, and prognosis of COVID-19. ODN2088 is a murine TLR9 antagonist that has been shown to be potent in preventing hepatic inflammation and fibrosis in a CCl4-induced liver fibrosis mouse model [23,33]. In this study, we evaluated ODN2088 as a potential therapeutic agent for prevention of pulmonary injury, inflammation, and fibrosis.

Apoptosis has a vital role in initiating and sustaining fibrosis during the pathophysiology of interstitial lung disease [41]. The pathogenic mechanisms of bleomycin-induced lung fibrosis and idiopathic pulmonary fibrosis (IPF) involve induction of alveolar epithelial cell apoptosis through either the mitochondrial pathway or the caspase cascade linked to the Fas-FasL pathway [ 42 , 43]. Our results showed that ODN2088 greatly decreased the proportion of apoptotic bronchial cells, endothelial cells, and alveolar pneumocytes in bleomycin-treated mice, as represented by low cleaved caspase- 3 immunostaining.

Recent studies attributed the lung fibrosis seen in COVID-19 cases to an excessive inflammatory response [44]. TNF- $\alpha$, an important mediator of inflammation, triggers TGF- $\beta 1$ and nuclear factor- $\mathrm{\kappa B}$ and enhances proliferation and fibroblast differentiation, which ultimately lead to pulmonary fibrosis $[45,46]$. Previous research showed that a TNF- $\alpha$ antagonist alleviated bleomycin-induced pulmonary fibrosis in mice [47]. In this context, we evaluated ODN2088 as an anti-inflammatory agent against bleomycin-induced pneumonia. In this study, ODN2088 miti- gated the inflammatory response in injured lungs, as evidenced by low TNF- $\alpha$ expression and a mild inflammatory response in the pulmonary tissues of BLEO/ODN2088-treated mice.

Prior studies emphasized that TLR9 is upregulated in IPF lungs, and CpG-ODN (a TLR agonist) promotes the differentiation of IPF-derived lung fibroblasts into myofibroblasts in vitro [48]. In addition, CpG-ODN was found to induce renal interstitial fibrosis in a mouse model of lupus nephritis [49]. Consistent with these previous studies, the present study showed the absence of pulmonary myofibroblasts in ODN2088-treated lung tissues compared to bleomycin-treated ones. Moreover, $\alpha$-smooth actin immunostaining and Van Gieson staining were used to confirm the inhibition of myofibroblast differentiation and subsequent fibrosis. The $\alpha$-smooth muscle actin expression level and the proportion of Van Gieson-stained collagen fibers were significantly lower in the lungs of BLEO/ODN2088-treated mice compared to those of bleomycin-treated mice. All these findings demonstrate that ODN2088 directly inhibits myofibroblast differentiation and development of pulmonary fibrosis induced by bleomycin.

Furthermore, our in vivo study showed a significant ameliorative effect in the lungs of mice given ODN2088 and bleomycin, as evidenced by a decrease in hemodynamic pulmonary lesions caused by bleomycin, such as microvascular damage, pulmonary edema, thrombosis, and hemorrhage. In coronavirus-infected patients, diffuse alveolar damage with serious pulmonary thrombosis and vascular injury has been documented [50], indicating that targeting TLR9 can improve the clinical presentation of ARDS, seen in some COVID-19 patients.

In conclusion, our study demonstrated that ODN2088 was potent in prevention of bleomycin-induced pulmonary injury, inflammation, and fibrosis. ODN2088 markedly diminished alveolar and vascular damages in pulmonary tissues and decreased the production of pro-inflammatory cytokines, inflammatory cellular aggregations, and myofibroblastic proliferation. These findings indicate that blocking TLR9 could be beneficial to treatment of severe acute respiratory syndrome, Middle East respiratory syndrome, and COVID-19 as a novel therapeutic modality to prevent pulmonary injury, fibrosis, and associated hemodynamic abnormalities, especially in the early stages.

\section{Ethics Statement}

All animal work was approved by the Bioethics Committee of Benha University. All in vivo experiments were conducted after the approved by Animal Care and Use Committee of Benha University. Animal welfare and comfort was emphasized during the study. All authors have agreed to publish this manuscript. 


\section{Availability of Data and Material}

All data generated or analyzed during the study are included in this published article.

\section{Code Availability}

Not applicable.

\section{ORCID}

Badr Alzahrani Mohamed M. S. Gaballa Ahmed A. Tantawy

Maha A. Moussa

Salma A. Shoulah Said M. Elshafae

https://orcid.org/0000-0003-4158-640X https://orcid.org/0000-0003-2949-430X https://orcid.org/0000-0002-5017-686X https://orcid.org/0000-0002-6987-9012 https://orcid.org/0000-0003-2115-1572 https://orcid.org/0000-0001-6285-5483

\section{Author Contributions}

Conceptualization: BA, AAT, MMSG, SME. Data curation: AAT, MMSG SME. Formal analysis: BA, MMSG, AAT, MAM, SAS, SME. Funding acquistion: BA, AAT. Investigation: BA, AAT, MMSG, SME. Methodology: AAT, MMSG, SME. Supervision: AAT, SME. Validation: BA, AAT, MMSG, SME, MAM, SAS. Writing_orginal draft: BA, MMSG. Writing-review \& editing: AAT, SME. Approval of final manuscript: all authors.

\section{Conflicts of Interest}

The authors declare that they have no potential conflicts of interest.

\section{Funding Statement}

No funding to declare.

\section{References}

1. She J, Jiang J, Ye L, Hu L, Bai C, Song Y. 2019 novel coronavirus of pneumonia in Wuhan, China: emerging attack and management strategies. Clin Transl Med 2020; 9: 19.

2. Wu Z, McGoogan JM. Characteristics of and important lessons from the coronavirus disease 2019 (COVID-19) outbreak in China: summary of a report of 72314 cases from the Chinese Center for Disease Control and Prevention. JAMA 2020; 323: 1239-42.

3. Matthay MA, Zemans RL. The acute respiratory distress syndrome: pathogenesis and treatment. Annu Rev Pathol 2011; 6: 147-63.

4. Moore BB, Hogaboam CM. Murine models of pulmonary fibrosis. Am J Physiol Lung Cell Mol Physiol 2008; 294: L152-60.

5. Moroni M, Giannetta L, Gelosa G, et al. Second-line chemotherapy with bleomycin, methotrexate, and vinorelbine (BMV) for patients with squamous cell carcinoma of the head, neck and esophagus (SCC-HN\&E) pretreated with a cisplatin-containing regimen: a phase II study. J Chemother 2003; 15: 394-9.

6. Canellos GP. Lymphoma: present and future challenges. Semin Hematol 2004; 41(4 Suppl 7): 26-31.

7. Rimmer Y, Chester J, Joffe J, et al. Accelerated BEP: a phase I trial of dose-dense BEP for intermediate and poor prognosis metastatic germ cell tumour. Br J Cancer 2011; 105: 766-72.

8. Gasse P, Riteau N, Charron S, et al. Uric acid is a danger signal activating NALP3 inflammasome in lung injury inflammation and fibrosis. Am J Respir Crit Care Med 2009; 179: 903-13.

9. Ruscitti F, Ravanetti F, Essers J, et al. Longitudinal assessment of bleomycin-induced lung fibrosis by Micro-CT correlates with histological evaluation in mice. Multidiscip Respir Med 2017; 12: 8.

10. Moore BB, Lawson WE, Oury TD, Sisson TH, Raghavendran K,
Hogaboam CM. Animal models of fibrotic lung disease. Am J Respir Cell Mol Biol 2013; 49: 167-79.

11. Imai Y, Kuba K, Neely GG, et al. Identification of oxidative stress and Toll-like receptor 4 signaling as a key pathway of acute lung injury. Cell 2008; 133: 235-49.

12. Lafferty EI, Qureshi ST, Schnare M. The role of toll-like receptors in acute and chronic lung inflammation. J Inflamm (Lond) 2010; 7: 57.

13. Arancibia SA, Beltran CJ, Aguirre IM, et al. Toll-like receptors are key participants in innate immune responses. Biol Res 2007; 40: 97112.

14. Gao W, Xiong Y, Li Q, Yang H. Inhibition of Toll-like receptor signaling as a promising therapy for inflammatory diseases: a journey from molecular to nano therapeutics. Front Physiol 2017; 8: 508.

15. Knuefermann P, Baumgarten G, Koch A, et al. CpG oligonucleotide activates Toll-like receptor 9 and causes lung inflammation in vivo. Respir Res 2007; 8: 72.

16. Tasaka S, Kamata H, Miyamoto K, et al. Intratracheal synthetic CpG oligodeoxynucleotide causes acute lung injury with systemic inflammatory response. Respir Res 2009; 10: 84.

17. Huang L, Chang W, Huang Y, Xu X, Yang Y, Qiu H. Prognostic value of plasma mitochondrial DNA in acute respiratory distress syndrome (ARDS): a single-center observational study. J Thorac Dis 2020; $12: 1320-8$.

18. Faust HE, Reilly JP, Anderson BJ, et al. Plasma mitochondrial DNA levels are associated with ARDS in trauma and sepsis patients. Chest 2020; 157: 67-76.

19. Koupenova M, Mick E, Mikhalev E, Benjamin EJ, Tanriverdi K, Freedman JE. Sex differences in platelet toll-like receptors and their association with cardiovascular risk factors. Arterioscler Thromb Vasc Biol 2015; 35: 1030-7.

20. El Kebir D, Damlaj A, Makhezer N, Filep JG. Toll-like receptor 9 signaling regulates tissue factor and tissue factor pathway inhibitor expression in human endothelial cells and coagulation in mice. Crit Care Med 2015; 43: e179-89.

21. Hemmi H, Takeuchi O, Kawai T, et al. A Toll-like receptor recognizes bacterial DNA. Nature 2000; 408: 740-5.

22. Jurk M, Vollmer J. Therapeutic applications of synthetic CpG oligodeoxynucleotides as TLR9 agonists for immune modulation. BioDrugs 2007; 21: 387-401.

23. Alzahrani B. Hepatoprotective impact of TLR9 antagonist ODN 2088 against carbon tetrachloride (CCl4)-induced hepatic injury. Am J Biochem Biotechnol 2020; 16: 9-14.

24. Akishima Y, Akasaka Y, Ishikawa Y, et al. Role of macrophage and smooth muscle cell apoptosis in association with oxidized low-density lipoprotein in the atherosclerotic development. Mod Pathol 2005; 18: 365-73.

25. Wang J, Wang BJ, Yang JC, et al. Research advances in the mechanism of pulmonary fibrosis induced by coronavirus disease 2019 and the corresponding therapeutic measures. Zhonghua Shao Shang Za Zhi 2020; 36: 691-7.

26. Moeller A, Gilpin SE, Ask K, et al. Circulating fibrocytes are an indicator of poor prognosis in idiopathic pulmonary fibrosis. Am J Respir Crit Care Med 2009; 179: 588-94.

27. Liu T, De Los Santos FG, Phan SH. The bleomycin model of pulmonary fibrosis. Methods Mol Biol 2017; 1627: 27-42.

28. Amin M, Wu V, Odish M, et al. A case of severe ARDS due to bleomycin-induced lung inury requiring ECMO support. Chest 2020; 158(4 Suppl): A1126-7. 
29. Daphale A, Acharya S, Shukla S, Alegaonkar S. Bleomycin induced acute respiratory distress syndrome. J Case Rep 2017; 7: 55-7.

30. Wilson MS, Wynn TA. Pulmonary fibrosis: pathogenesis, etiology and regulation. Mucosal Immunol 2009; 2: 103-21.

31. Noble PW, Barkauskas CE, Jiang D. Pulmonary fibrosis: patterns and perpetrators. J Clin Invest 2012; 122: 2756-62.

32. Moeller A, Ask K, Warburton D, Gauldie J, Kolb M. The bleomycin animal model: a useful tool to investigate treatment options for idiopathic pulmonary fibrosis? Int J Biochem Cell Biol 2008; 40: 362-82.

33. Alzahrani B, A AMA, Tantawy A. Therapeutic impact of ODN2088 to block TLR9 activity in induced liver fibrosis mice. Pak J Biol Sci 2021; 24: 122-31.

34. Chaudhary NI, Schnapp A, Park JE. Pharmacologic differentiation of inflammation and fibrosis in the rat bleomycin model. Am J Respir Crit Care Med 2006; 173: 769-76.

35. Chandler DB. Possible mechanisms of bleomycin-induced fibrosis. Clin Chest Med 1990; 11: 21-30.

36. Reinert T, da Rocha Baldotto CS, Nunes FA, de Souza Scheliga AA. Bleomycin-induced lung injury. J Cancer Res 2013; 2013: 480608.

37. Williamson JD, Sadofsky LR, Hart SP. The pathogenesis of bleomycin-induced lung injury in animals and its applicability to human idiopathic pulmonary fibrosis. Exp Lung Res 2015; 41: 57-73.

38. Khanmohammadi S, Rezaei N. Role of Toll-like receptors in the pathogenesis of COVID-19. J Med Virol 2021; 93: 2735-9.

39. Lin L, Luo S, Qin R, et al. Long-term infection of SARS-CoV-2 changed the body's immune status. Clin Immunol 2020; 218: 108524.

40. He L, Ding Y, Zhang Q, et al. Expression of elevated levels of pro-inflammatory cytokines in SARS-CoV-infected ACE2+ cells in SARS patients: relation to the acute lung injury and pathogenesis of SARS. J Pathol 2006; 210: 288-97.

41. Ellson CD, Dunmore R, Hogaboam CM, Sleeman MA, Murray
LA. Danger-associated molecular patterns and danger signals in idiopathic pulmonary fibrosis. Am J Respir Cell Mol Biol 2014; 51: 163-8.

42. Kuwano K, Kunitake R, Maeyama T, et al. Attenuation of bleomycininduced pneumopathy in mice by a caspase inhibitor. Am J Physiol Lung Cell Mol Physiol 2001; 280: L316-25.

43. Gazdhar A, Fachinger P, van Leer C, et al. Gene transfer of hepatocyte growth factor by electroporation reduces bleomycin-induced lung fibrosis. Am J Physiol Lung Cell Mol Physiol 2007; 292: L52936.

44. Garcia-Revilla J, Deierborg T, Venero JL, Boza-Serrano A. Hyperinflammation and fibrosis in severe COVID-19 patients: galectin-3, a target molecule to consider. Front Immunol 2020; 11: 2069.

45. Della Latta V, Cecchettini A, Del Ry S, Morales MA. Bleomycin in the setting of lung fibrosis induction: from biological mechanisms to counteractions. Pharmacol Res 2015; 97: 122-30.

46. Zhao Y, Tian B, Sadygov RG, Zhang Y, Brasier AR. Integrative proteomic analysis reveals reprograming tumor necrosis factor signaling in epithelial mesenchymal transition. J Proteomics 2016; 148: 126-38.

47. Altintas N, Erboga M, Aktas C, et al. Protective effect of infliximab, a tumor necrosis factor-alfa inhibitor, on bleomycin-induced lung fibrosis in rats. Inflammation 2016; 39: 65-78.

48. Hinz B. Formation and function of the myofibroblast during tissue repair. J Invest Dermatol 2007; 127: 526-37.

49. Anders HJ, Vielhauer V, Eis V, et al. Activation of toll-like receptor-9 induces progression of renal disease in MRL-Fas(lpr) mice. FASEB J 2004; 18: 534-6.

50. Angeles Montero-Fernandez M, Pardo-Garcia R. Histopathology features of the lung in COVID-19 patients. Diagn Histopathol (Oxf) 2021; 27: 123-7. 